\title{
Wireless Sensing Node Deployment of Coal Mine Based on the Probability Sensor Model
}

\author{
Ya Wang \\ College of Electrical and Information Engineering, Anhui \\ University of Science and Technology, Huainan, China \\ School of Computer and Information, Fuyang Teachers \\ College, Fuyang, China \\ E-mail:fync_wy80@163.com
}

\author{
Meng-Ran Zhou \\ College of Electrical and Information Engineering, Anhui \\ University of Science and Technology, Huainan, China \\ E-mail:fync_wy80@163.com
}

\begin{abstract}
The wireless sensor networks (WSN) with the distributed network protocol, the node with the abilities of sensor, wireless communications and data processing. It can monitor, aware and acquire the information of environment or monitoring object in real time. The coal mine can overcome the difficulties using the wireless sensor networks, and realize its coal mining, boring work surface, goaf gas, staff orientation such as wireless access detection. After the disaster, WSN can quickly reconstruct the environmental monitoring system to enhance the coal mine safety surveillance system in disaster conditions remaining. This paper analyses the characteristics of narrow space in the coal mine laneway, gives the probability sensor model (PSM) for laneway narrow space, analyses the relationships among perceived probability, effective awareness radius and other sensing parameters. For the semi-enclosed narrow space, to meet the maximum coverage and effective redundancy, it gives the node deployment scenarios in laneway, to lay the foundation for reliability research of wireless sensor networks in coal mine underground.
\end{abstract}

Keywords- Coal mine; the probability sensor model (PSM); semi-enclosed narrow space; node deployment

\section{INTRODUCTION}

In order to ensure the issue of coal mine safe production, a wide variety of automatic systems such as security and production monitoring systems have been gradually established in mining process, for the miner, equipment and environmental disasters such as safety information for monitoring, and controlling[1]. The challenging environments in the underground coal mine where the natural conditions are complex and poor. The cabling is not readily and the existing wired communications network cannot reach some security key areas, in key areas such as goaf area and work surface. It is difficult to fully monitor and control many security risks.

In recent years, with the emergence of technology internet of things (IoT), IoT provides the new methods for coal mine safe production, monitoring and early warning of disasters [2]. The sensor technology as an important technology in IoT, promotes the development of wireless sensor networks. The wireless sensor networks has the distributed network protocol, its node which has the abilities of sensor, wireless communications and data processing. It can monitor, perceive and acquire the information of environment or monitoring object in real time.

Using the wireless sensor networks, the coal mine can overcome the difficulties, and realize its coal mining, boring work surface, goaf gas, staff orientation such as wireless access detection. After the disaster, WSN can quickly reconstruct the environmental monitoring system to enhance the coal mine safety surveillance system in disaster conditions remaining [3].

As a result of coal mine where geological conditions are complex, the nodes generally deployed in unattended or hazardous areas. The energy of nodes is consumed too quickly in disaster, or accidents cause a part of wireless sensing nodes fail suddenly. As the coverage of nodes in laneway is inadequate, and the capacity of network data transmission seriously degraded, so that could not assist rescue workers to complete the disaster control and rescue work [4-5].Therefore, in the application of large-scale WSN in coal mine, for the narrow space, how to establish the suitable probability of sensor models for the practical application environment and complete sense node deployment issues.

For the issues about wireless sensing node deployment, the current research is mainly concentrated in the 2dimensional plane, this paper mainly focus on the coal mine where is the semi-enclosed 3-dimensional space. In this paper, the related probability sensor model of the wireless sensor networks is built, node deployment scenarios are established, and the appropriate topology control and emulation are completed in the MATLAB.

\section{THE NARROW SPACE}

The surface of coal mine laneway which is covered with coal and rock layer is rough, providing corridor for pedestrian and transport in laneway. Constraining by the mine underground space, the laneway is a semi-enclosed narrow space, with a tree branching structures. The Wireless sensor networks can not only implement the environmental monitoring, the electrical and mechanical monitoring, staff and equipment tracking tasks, but also play its important role in emergency rescue work such as the laneway landslides, coal mine gas explosion and water inrush. It has a very wide application prospect in the narrow and long space [6].

The laneway has two walls and ceiling which common shape of laneway is the round vault 3-dimensional or 
rectangular space, which cross-section as a rectangle or an approximate rectangle. The defense-in-distance is far larger than the width and height of the cross-section. According to some common laneway length, breadth and height draws the narrow space, shown as Figure 1. The breadth (B) of laneway is approximate $5-6 \mathrm{~m}$, height $(\mathrm{H})$ is approximate 3$4 \mathrm{~m}$, and length (L) is approximate a few hundred meters, a few kilometers, even several tens of kilometers. In Figure 1, the 100 meters laneway is taken as a research object.

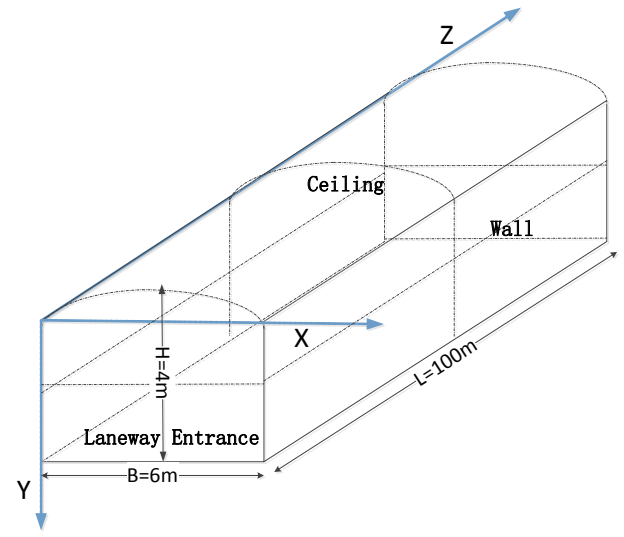

Figure 1. The narrow space model of underground laneway.

Restricted by long and narrow geographical environment, the wireless communications environment of underground coal mine is poor, wireless node signal transmission exists multi-path fading and wireless signal transmission distance is short. In order to ensure reliable data communications, if the high-coverage rate is used to decorate the sensing node, there are a large number of nodes in laneway. The communications among nodes is too prone to interference and channel competition. The data transport is reliably poor, resulting in sensor layer network topology dynamical change. Therefore, according to the actual situation of the long and narrow space, the characteristics of sensor nodes carry out a rational and effective deployment of nodes.

\section{THE PROBABILITY SENSOR MODDEL}

In wireless sensor networks, the node sensing model can be divided into the binary (0-1) model and probability model [7]. The node can only control what had happened in the sphere where the node is at sphere center and the perception range is the radius of the sphere. The main difference between the two models is that, in the range of perception, the probability of event which was correct perceived is not a constant. In practical application scenario, particularly the long and narrow semi-enclosed space, the nodes have low reliability and harsh environmental conditions. The binary model cannot obviously accurately describe network coverage capabilities. The detection capabilities of sensor nodes are uncertain because of the interference of environment noise and the attenuation of signal intensity with transmission distance. However, the probabilistic sensor model reflects the uncertainty. The sensor model converts the physical sensing signal to the probability value, which represents the confidence level of the information collected by the sensor.

For the probability sensor model in long and narrow space, the model is represented by the following model. Assume sensor $\mathrm{S}_{i}$ is deployed at point $\left(x_{i}, y_{i}, z_{i}\right)$. For any point $\mathrm{P}_{\text {at }}(x, y, z)$, we denote the Euclidean distance between ${ }^{\mathrm{S}_{i}}$ and $\mathrm{P}$ as

$$
d\left(s_{i}, P\right)=\sqrt{\left(x_{i}-x\right)^{2}+\left(y_{i}-y\right)^{2}+\left(z_{i}-z\right)^{2}} \text {. Equation }
$$

(1) shows the probability model the expresses the coverage, define the node's coverage perceived probability cc that can be expressed as follows:

$$
C\left(S_{\mathrm{i}}, P\right)= \begin{cases}0 & r+r_{e}<d\left(s_{i}, P\right) \leq R \\ e^{-\alpha\left(d\left(s_{i}, P\right)-\left(\mathrm{r}-\mathrm{r}_{e}\right)\right)} & r-r_{e}<d\left(s_{i}, P\right) \leq r+r_{e} \\ 1 & d\left(s_{i}, P\right) \leq r-r_{e}\end{cases}
$$

Where $R$ is each sensor maximum sensor radius (the physical parameters), $r$ is the range the radius of identifying region (i.e. the node detection range), $r_{e}\left(r_{e}<r\right)$ a measure of the uncertainty in sensor detection, $\alpha(\alpha>0)$ is parameter that measure detection probability which the target is properly monitored by sensor nodes, which the decrease with increasing distance from the speed. Parameters

$r_{e}$ and $\alpha$ determines the wireless sensor nodes for the Identity attribute.
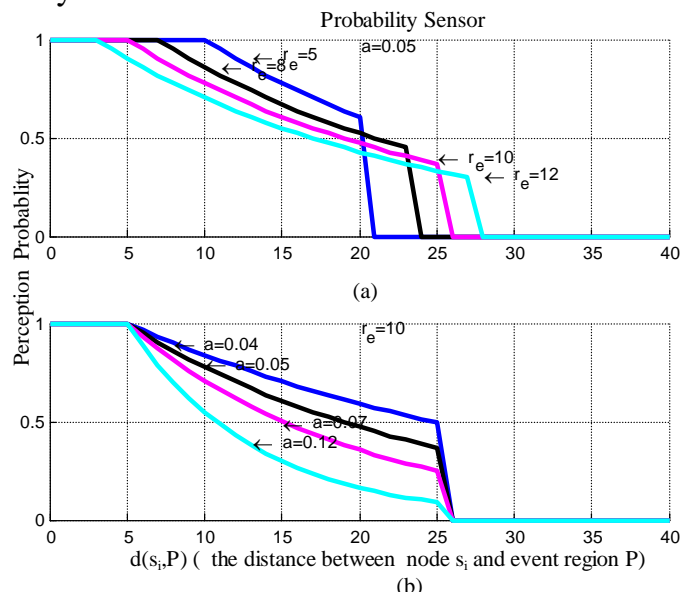

Figure 2. Probability sensor model (a) Different ${ }^{r_{e}}$ (b) Different $\alpha$.

In Figure 2.(a), when the sensor nodes properly monitors the events, given parameter $\alpha$, the node detection areas is gradually increasing, adjusting the sensor nodes determine detection capabilities $r_{e}$, as the increasing of the 
distance $d\left(s_{i}, P\right)$. In Figure 2 (b), given parameter $r_{e}$, attaching the sense node detection capacity, as increasing of $\alpha$, the rate of change about perceived probability also gradually get faster.

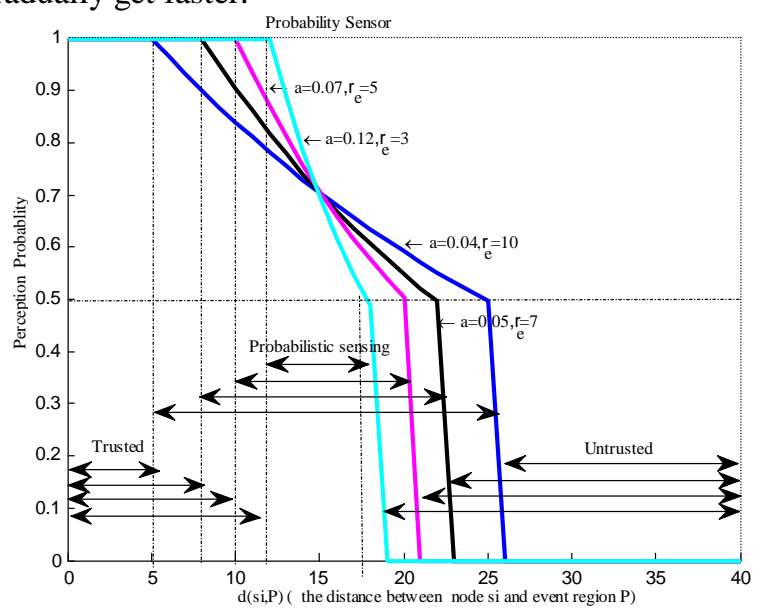

Figure 3. The various sensing probability regions of sensor nodes.

In Figure 3, the sensor distance critical threshold in the trusted region is $r-r_{e}$, as the increasing of the distance $d\left(s_{i}, P\right)$, the perceived probability node move from the trusted region to the probabilistic sensing region. When the perceived probability drops to below 0.5 , the region is untrusted. The sensor distance critical threshold in the untrusted region is $r+r_{e}$. The following relationships $0<r-r_{e}<r+r_{e}<R$ exist in probability sensor model, where the value of parameters $r, r_{e}$ and $\alpha$ is determined by the characteristics of the sensor itself and monitoring object.

The derivation process of the maximum effective sense radius of long and narrow space sensing nodes is given:

$$
\begin{aligned}
& \text { When } C(s, P)=e^{-\alpha\left(d(s, P)-\left(\mathrm{r}-\mathrm{r}_{e}\right)\right)}=0.5 \\
& \Rightarrow e^{-\alpha\left(d(s, P)-\left(\mathrm{r}-\mathrm{r}_{e}\right)\right)}=e^{\ln 0.5} \\
& \Rightarrow-\alpha\left(d(s, P)-\left(r-r_{e}\right)\right)=\ln 0.5 \\
& \Rightarrow d(s, P)=\left(r-r_{e}\right)-\alpha^{-1} \ln 0.5 \\
& \Rightarrow d(s, P)=\left(r-r_{e}\right)+0.693 \alpha^{-1}
\end{aligned}
$$$$
\text { Where }\left(r-r_{e}\right)+0.693 \alpha^{-1} \text { can be defined the }
$$

maximum effective sense radius $r_{s}$ (It is equal to the distance critical threshold of untrusted region).

It can be inferred from the above that, in the process of node deployment, the distance between the node and the event region is maintained in the maximum effective radius, which can satisfy the maximum probability of perception of the event.

\section{NODE DEPLOYMENT SCENARIOS}

The coverage of nodes is taken into node deployment scenario. Through the distribution of spatial location of sensor nodes in the network to adjust the implementation of the perception of physical information about region and object that is monitored.

The coverage of the network sensing region is the basis of availability and survivability [8]. Due to the limitations of natural conditions of underground laneway in coal mine, (1) the cross sectional area is small, and the utilization of node regional coverage is low (2) ceiling common exist micro-arc shape, in order to simplify research conditions, it is considered that the cross section is a rectangle (H-height and B-breadth) (3) the test region on the Z-axis length that is much larger than the effective sensing radius of the node. According to the network topology control in advance, the sensor node density to the key region is increased, that is the certainty coverage [9].For the complex mine environment, the coverage of the monitoring region is often less than the maximum effective coverage of the sensor itself. The ratio of the actual coverage and maximum effective coverage is defined the effective coverage. In the laneway which is similar to the rectangle, the sensing radius and the breadth and height of the long and narrow space determine the actual coverage volume. Restricting by a long and narrow semienclosed space, a large number of static nodes are needed to be arranged on both sides of laneway and ceiling to form a mesh model.

In accordance with the reference [6] proved to be seen, in order to make the coverage capability of the long and narrow space network optimal, in breadth (X-axis) and height (Yaxis) direction, it is needed to meet the requirements of maximum effective coverage and redundant coverage. In the long and narrow space, the nodes are deployed in the maximum coverage position to maximize the utilization of the node. When the ceiling nodes are deployed in the breadth of $\mathrm{B} / 2$, sensor nodes can achieve maximum coverage rate. When the height of the nodes is located at $\mathrm{H} / 2$, the effective coverage rate of the sensor nodes is the maximum.

Therefore, in the Figure 1, deployed within the laneway in the following way, given the breadth, height and length is respective $\mathrm{B}, \mathrm{H}$ and $\mathrm{L}$, the effective sensing radius $r_{S}$.There are three points in laneway space, ceiling point $A_{0}(B / 2,0,0)$,the points in two sides $A_{1}\left(B, 0, Z_{1}\right)$ and $A_{2}\left(\mathrm{~B}, H / 2, Z_{2}\right)$, composing a space triangle $\Delta A_{0} A_{1} A_{2}$. The distance between nodes of ceiling and the side wall is less than the effective sensing radius ${ }^{r}$, as shown the following inequality: 


$$
\left\{\begin{array}{c}
A_{0} A_{1}=\sqrt{(B-B / 2)^{2}+0+z_{1}^{2}} \leq r_{s} \\
A_{1} A_{2}=\sqrt{(B-\mathrm{B})^{2}+(0-H / 2)^{2}+\left(z_{1}-z_{2}\right)^{2}} \leq r_{s} \\
A_{0} A_{2}=\sqrt{(B / 2-B)^{2}+(0-H / 2)^{2}+z_{2}^{2}} \leq r_{s}
\end{array}\right.
$$

By the above inequality, can derive that the value of $Z_{1}$ and $Z_{2}$ are the $r_{s}$ and ${ }^{r_{s}}-1$.In the 3-dimensional laneway space, nodes can form a number of similar spatial triangles, with deployment of laneway narrow space in accordance with the way in order to meet the effective coverage redundancy.

When the maximum effective sensing radius $r_{s}$ approximately $10 \mathrm{~m}$, in $\mathrm{B}=6 \mathrm{~m}, \mathrm{~L}=100 \mathrm{~m}$ and $\mathrm{H}=4 \mathrm{~m}$ laneway space, the deployment location of nodes as shown in Figure 4:

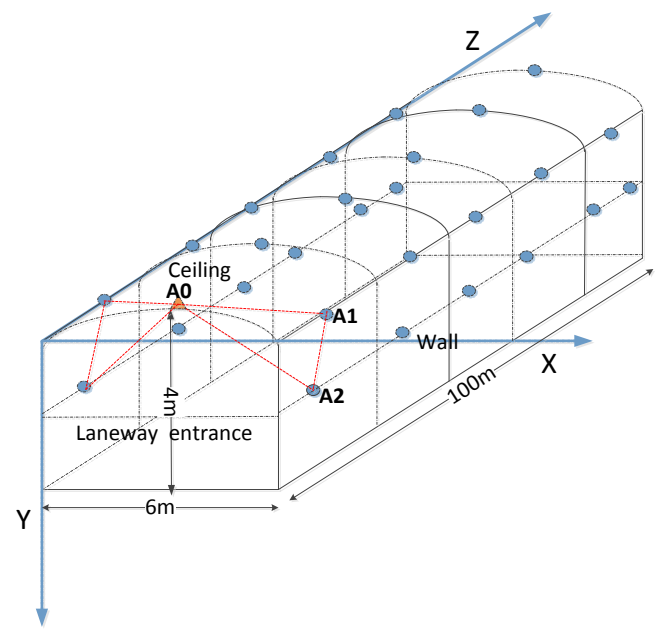

Figure 4. Nodes deployment in long and narrow laneway space.

\section{CONCLUSION}

In order to adapt to the complex environment of underground coal mine, with certainty how to override node deployment, established the probability perception model of long and narrow space, given the relationship among the perceived probability, the effective sensing radius and the characteristic parameters. According to the effective sensing radius, the 3-dimensional space node deployment scenario is constructed to meet the effective coverage and redundancy.

\section{ACKNOWLEDGMENT}

This research was supported under the project "Twelfth Five-year" National Science and Technology Support Projects (2013BAK06B01), the National Science Foundation Project of China (51174258), the National Safe Production Critical Incident to Key Technologies Science and Technology Project (anhui-0001-2016AQ), the Provincial Natural Science Research Project (KJ2015A278, KJ2015A295) and the College Young Talent Support Plan ( gxyqzd2016194).

\section{REFERENCES}

[1] SUN Yanjing, QIAN Jiansheng, LI Shiyin, DOU Linming. "Theory and Key Technology for Mine Internet of Tings" . Coal Science and Technology, 2011, vol.39(2),pp. 69-72. (in Chinese)

[2] YANG Li. "Development direction of China coal science and technology during the "Twelfth Five-Year Plan"'. Clean Coal Technology, 2013, vol.19(1),pp. 112-114. (in Chinese)

[3] SUN Yanjing, ZUO Haiwei, QIAN Jiansheng, ZHAO Fuyin. "Application Mode and Key Technology of Internet of Things Faced to Coal Mine Safety Production “. Coal Science and Technology, 2013, vol.41(1), pp. 84-88. (in Chinese)

[4] Zhang GuoPeng, Liu Peng, Ding Enjie. "Study on Topology Reconstruction and Data Transmission of Mine Emergency Communication Network". Coal Science and Technology, 2015, vol.43(11), pp. 95-100. (in Chinese)

[5] Sun Chao. "Study on Energy-Saving and Fault-Tolerant Algorithms for Wireless Sensor Networks Based on Topology Control" .Hebei: Yanshan University, 2010. (in Chinese)

[6] Zhou GongBo. "The Reliability Supported Technology of Wireless Sensor Networks in Long-narrow Region”.Beijing: China University of Mining and Technology, 2010. (in Chinese)

[7] Zou y Chakrabarty-K. . "Sensor deployment and target localization based on virtual forces".IEEE INFOCOM. Institute of Electrical Engineers Inc(IEEE), 2003, pp.1293-1303.

[8] Wang LiangMin, LiaoWenJian. "Research on the theory and technology of wireless sensor network" .Beijing: People's Posts and Telecommunications Press, 2011. (in Chinese)

[9] Ren Yan, Zhang SiDong,Zhang HongKe. "Theories and Algorithms of Coverage Control for Wireless Sensor Networks ". Journal of Software, 2006, vol.17(3) pp.422-433. (in Chinese) 